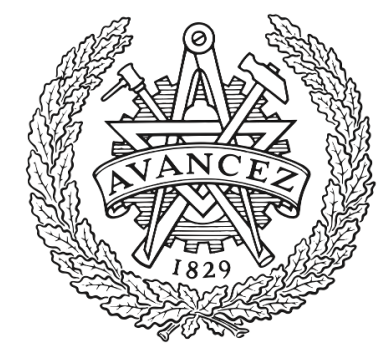

CHALMERS

UNIVERSITY OF TECHNOLOGY

\title{
A low phase noise W-band MMIC GaN HEMT oscillator
}

Downloaded from: https://research.chalmers.se, 2023-04-26 09:27 UTC

Citation for the original published paper (version of record):

Thanh, T., Yan, Y., Kuylenstierna, D. (2020). A low phase noise W-band MMIC GaN HEMT oscillator. Asia-Pacific Microwave Conference Proceedings, APMC: 115-115.

http://dx.doi.org/10.1109/APMC47863.2020.9331430

N.B. When citing this work, cite the original published paper. 


\section{A low phase noise W-band MMIC GaN HEMT oscillator}

\author{
Thanh Ngoc Thi Do \\ Department of Microtechnology and \\ Nanoscience \\ Chalmers University of Technology \\ Gothenburg, Sweden \\ tngoc@chalmers.se
}

\author{
Yu Yan \\ Department of Microtechnology and \\ Nanoscience \\ Chalmers University of Technology \\ Gothenburg, Sweden \\ yu.yan@chalmers.se
}

\author{
Dan Kuylenstierna \\ Department of Microtechnology and \\ Nanoscience \\ Chalmers University of Technology \\ Gothenburg, Sweden \\ dan.kuylenstierna@chalmers.se
}

\begin{abstract}
This paper presents a fundamental mode W-band MMIC balanced Colpitts oscillator implemented in an advanced $60 \mathrm{~nm}$ gallium nitride (GaN) high electron mobility transistor (HEMT) process from OMMIC foundry. The oscillator operates around $85 \mathrm{GHz}$ with measured peak output power of nearly 0 $\mathrm{dBm}$ and phase noise at $10 \mathrm{MHz}$ offset better than $-120 \mathrm{dBc} / \mathrm{Hz}$. To the best authors' knowledge, the phase noise is state-of-the-art value for W-band monolithic microwave integrated circuit (MMIC) GaN HEMT oscillators.
\end{abstract}

Keywords- oscillator, gallium nitride (GaN), HEMT, millimeter-wave, monolithic microwave integrated circuit (MMIC), phase noise, W-band (75-110 GHz).

\section{INTRODUCTION}

In recent years, high resolution millimeter-wave (mmwave) radars have attracted interest due to promising applications in transportation industry [1-2], e.g. in aircraft traffic control systems to enhance vision in adverse weather/environment, etc. In order to offer an excellent definition and a wide detection range, the key radar components are power amplifiers and signal sources that are able to deliver high power and have low far-carrier phase noise performance at mm-wave frequencies, respectively. Besides the high performance, a compact module being cost effective in volume production is also important and challenging. Among many semiconductor technologies, short-gate length monolithic microwave integrated circuit (MMIC) GaN HEMT technology is the most potential candidate with capability of delivering sufficient power at mm-wave frequencies for mm-wave radars thanks to its particularly high breakdown voltage [3]. Recently, commercial GaN HEMT process lines also offer costeffectiveness in volume production. Further, it is well known that GaN HEMT is excellent for power amplifiers and there is a need for fully integration. Therefore, the development of low phase noise, sufficient output power integrated mm-wave GaN HEMT oscillators for cost effective compact mm-wave radars is necessary.

In this work, we report a low phase noise W-band balanced Colpitts MMIC oscillator in an advanced $60 \mathrm{~nm}$ GaN HEMT technology from OMMIC. The achieved phase noise at $10 \mathrm{MHz}$ offset is better than $-120 \mathrm{dBc} / \mathrm{Hz}$ and this is so far the lowest phase noise reported for W-band MMIC GaN HEMT oscillators.

\section{OSCILLATOR DESIGN}

The oscillator is designed and realized in OMMIC's $60 \mathrm{~nm}$ gate length D006GH GaN HEMT process on Si substrate with a cut off frequency $\left(f_{T}\right)$ and maximum oscillation frequency $\left(f_{\max }\right)$ of 190 and $250 \mathrm{GHz}$ respectively. A two-finger HEMT device with a gate-finger width of $25 \mu \mathrm{m}$ that gives sufficient gain at W-band, is selected. Once the active device is chosen, the oscillator topology is to be selected. In this work, a common-gate balanced Colpitts topology, as seen in Fig. 1(a), which is previously demonstrated to offer good phase noise [4], is used. The balanced topology can also theoretically obtain $3 \mathrm{~dB}$ lower phase noise compared to the single-ended topology. Fig. 1(b) shows the chip photo of the designed oscillator.

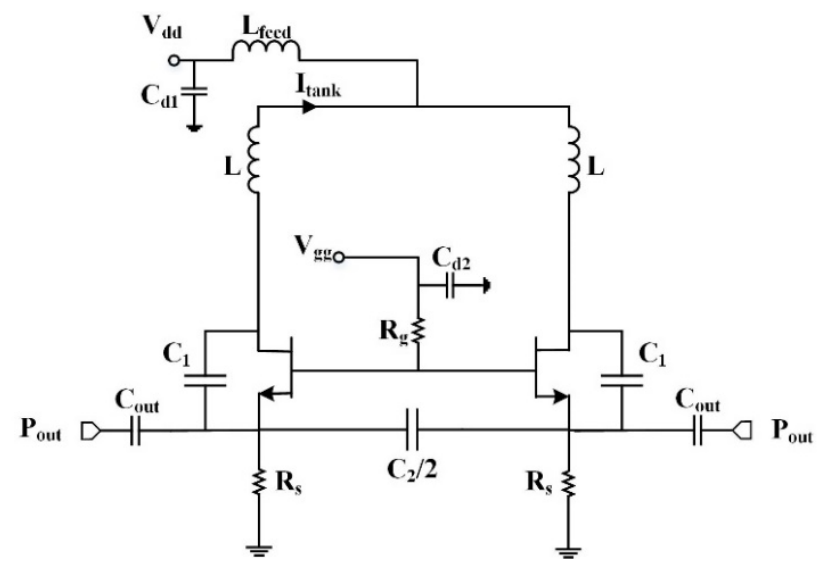

(a)

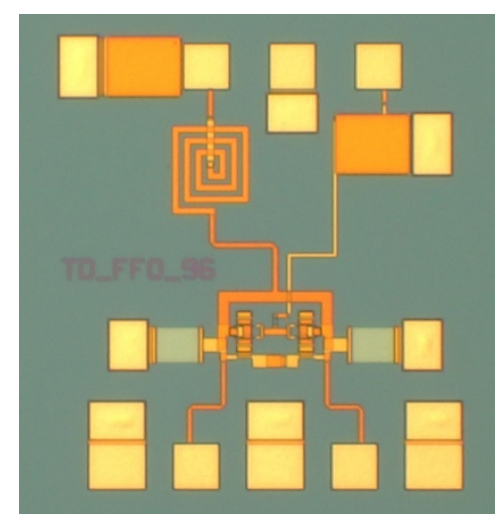

(b)

Fig. 1. Balanced Colpitts W-band GaN HEMT oscillator. (a) Schematic. (b) Chip photo. 
The oscillator is designed for sufficient output power and low phase noise at W-band. Thus, all component values in the schematic of the designed oscillator are optimized for that target. The tapping ratio of capacitive divider in Fig. 1(a) is 0.33 while the width of the microstrip line in the tank inductance is $20 \mu \mathrm{m}$. Since there is many parasitic components at high frequencies, the whole resonator is EMsimulated for the highest accuracy. Besides the design of the lumped-elements in the resonator, the bias networks also have to be designed. The gates are biased through a $1 \mathrm{k} \Omega$ resistor, while the drains are biased in the symmetry node of the tank inductor. The value of the source resistance is $50 \Omega$. The output signal are extracted through a MIM capacitance of 10 $\mathrm{fF}$ at both sides. The designed oscillator uses only one differential transistor pair without any additional combining networks, requiring less dc power consumption and therefore enabling higher power efficiency.

The circuit is designed using Harmonic Balance (HB) in Keysight's Advanced Design System (ADS 2017) with dedicated design kit. The size of the designed oscillator is $0.7 \times 0.9 \mathrm{~mm}^{2}$.

\section{MEASUREMENT RESULTS}

The designed oscillator is characterized using an FSUP50 signal-source analyzer from Rohde \& Schwarz. The low noise internal dc supplies of the FSUP are used for biasing the gate and the drain. The frequency range of the FSUP is extended using an external W-band sub harmonic mixer according to the measurement set-up in Fig. 7. The total loss from $\mathrm{W}$-band probe and coaxial connection is characterized to be $2 \mathrm{~dB}$.

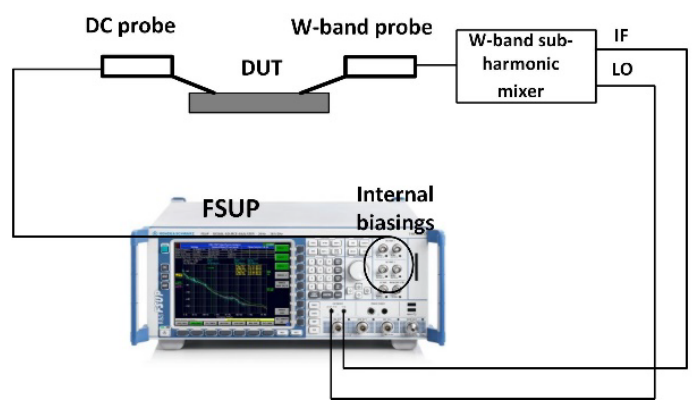

(a)

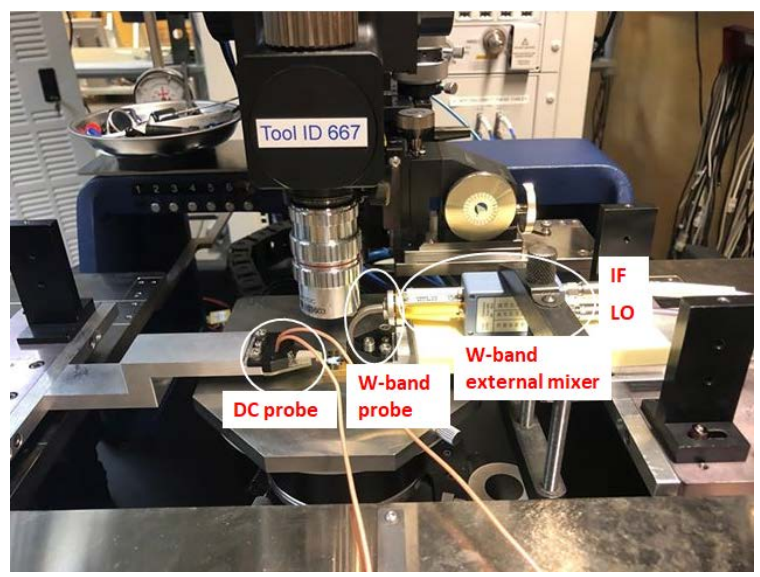

(b)

Fig. 2. Oscillator measurement setup. (a) Block diagram. (b) On-wafer test setup.

Fig. 3(a) shows the measured oscillating frequency versus gate bias voltage with drain bias voltage as a parameter.
Compared to the simulated oscillating frequency in HB simulation, i.e. $91 \mathrm{GHz}$, the measured oscillating frequency is shifted down around $8 \%$, likely due to miss-match of parasitic capacitance in the transistor model. Moreover, the measured dc current is also lower than the simulated dc current, likely due to a shift in pinch-voltage and a different compression characteristic.

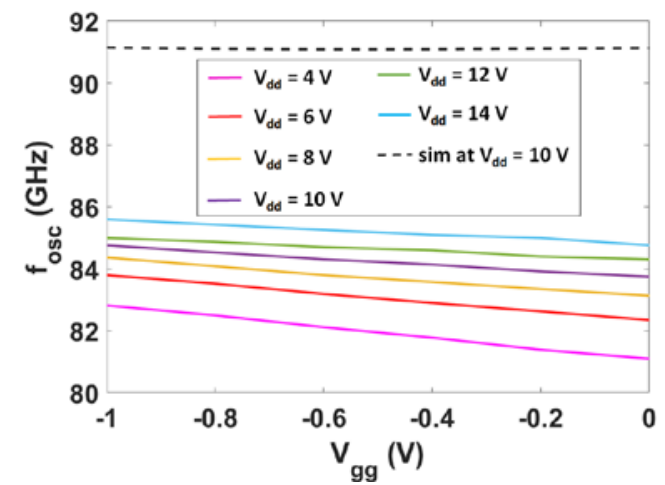

(a)

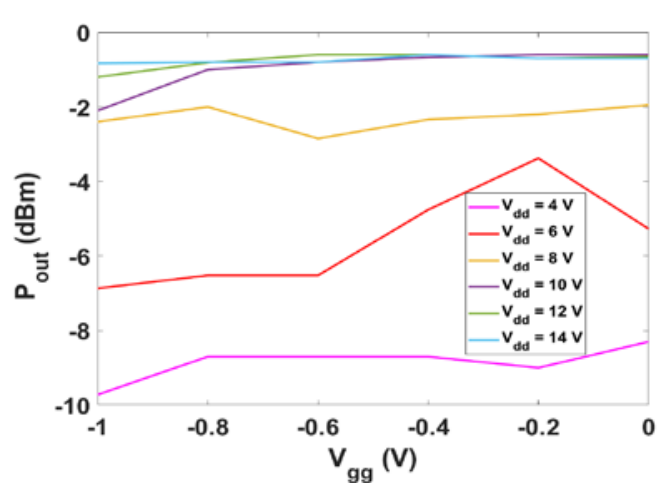

(b)

Fig. 3. The designed ocillator performance. (a) Oscillating frequency at different drain bias voltages versus the gate bias voltage. (b) Output power at different drain bias voltages versus the gate bias voltage .

Fig. 3(b) presents the measured RF output power of the designed oscillator versus gate bias voltage at different drain voltages after correcting the probe and connection loss. The measured output power is lower than the simulated one, e.g. for bias condition $V_{\mathrm{dd}}=10 \mathrm{~V} / V_{\mathrm{gg}}=-0.4 \mathrm{~V}$ the output power in measurement is about $-0.5 \mathrm{dBm}$ while the output power in HB simulation is about $2 \mathrm{dBm}$. This difference can be associated with different saturation characteristics, which was already identified from the dc current. By adjusting the gate bias voltage, a small bandwidth of $1 \mathrm{GHz}$ with moderate RF power can be achieved, e.g. at $V_{\mathrm{dd}}=10 \mathrm{~V}$. The dc power consumption varies from 32 to $616 \mathrm{~mW}$ versus different gate and drain bias voltages.

Accurate phase noise characterizations of free-running oscillators usually require some types of phase stabilization methods such as the PLL or the discriminator methods. The R\&S FSUP 50 signal source analyzer used in this work has a dedicated hardware option using cross-correlation of two PLLs to reach a very low noise floor [5]. Unfortunately, this method only works for free-running oscillators $<50 \mathrm{GHz}$ and not compatible with the external mixers needed to characterize the designed oscillator. Instead, phase noise in this work is determined by using the basic and simplest spectrum-analyzer method, i.e. reading directly from the 
measured spectrum. By manually adjusting frequency span and resolution bandwidth, phase noise is estimated from the measured spectrum.

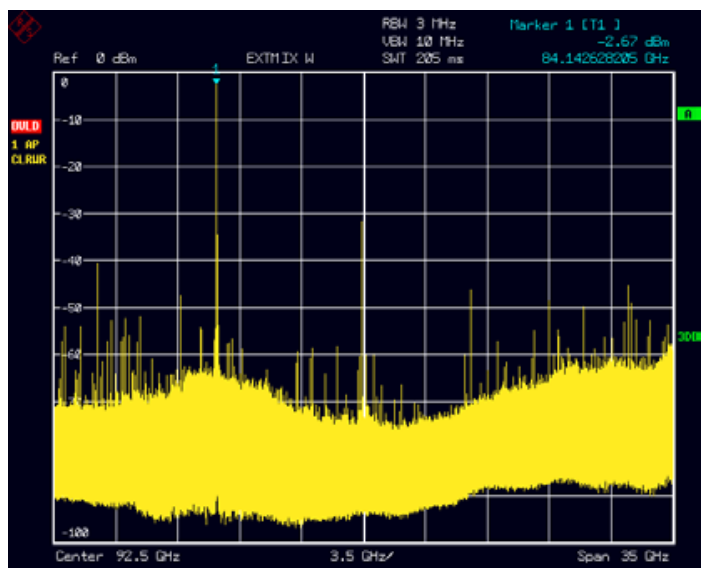

(a)

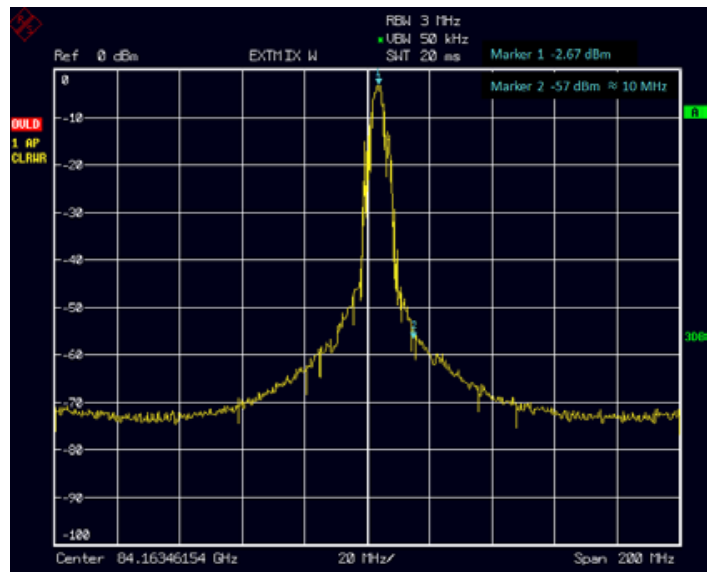

(b)

Fig. 4. Measured output spectrum from FSUP of the designed oscillator at $V_{\mathrm{dd}}=10 \mathrm{~V} / I_{\mathrm{dd}}=34 \mathrm{~mA}$. (a) in the whole W-band. (b) with frequency span of $200 \mathrm{MHz}$ (noted that the $2 \mathrm{~dB}$ probe and connection loss should be correct in the measured output power).

Fig. 4(a) and (b) show measured oscillator spectrum from FSUP for bias condition, $V_{\mathrm{dd}}=10 \mathrm{~V} / I_{\mathrm{dd}}=34 \mathrm{~mA}$ in the whole W-band and with the frequency span of $200 \mathrm{MHz}$, respectively. To calculate phase noise from the measured spectrum it is necessary to compensate for the used resolution bandwidth (RBW), which is $3 \mathrm{MHz}$ in Fig. 4. Single-sideband (SSB) phase noise $\mathcal{L}$ is defined as the normalized noise power within a $1 \mathrm{~Hz}$ bandwidth at an offset frequency ( $\Delta f)$. The phase noise is calculated as

$$
\mathcal{L}(\Delta f)=P_{n}(R B W)-P_{s}-10 \times \log _{10}(R B W \times \delta)
$$

where $P_{\mathrm{s}}$ is carrier signal power, $P_{\mathrm{n}}$ is the noise power within the resolution bandwidth (RBW) at offset $\Delta f$ and $\delta$ is a correction factor to correct the filters noise bandwidth and is equal to 1.128 that read from the datasheet of FSUP [5].

From the spectrum in Fig. 4(b) the measured phase noise at an off-set frequency of $10 \mathrm{MHz}$ can be estimated to about $-120 \mathrm{dBc} / \mathrm{Hz}$.
Table I compares the performance of millimeter-wave oscillators operating $>50 \mathrm{GHz}$ in GaN HEMT technology reported in open literature. They have different GaN HEMT processes and topologies. It is found that the presented oscillator obtains lowest phase noise.

TABLE I. COMPARISON WITH OTHER GAN HEMT OSCILLATORS OPERATING $>50 \mathrm{GHZ}$

\begin{tabular}{|c|c|c|c|c|c|c|}
\hline $\begin{array}{c}\mathbf{f}_{\text {osc }} \\
\mathbf{( G H z )}\end{array}$ & $\begin{array}{c}\mathbf{P}_{\text {out }} \\
\mathbf{( d B m )}\end{array}$ & $\begin{array}{c}\mathbf{B W} \\
\mathbf{( G H z )}\end{array}$ & $\begin{array}{c}\mathcal{L}(\mathbf{1 0 M H z}) \\
(\mathbf{d B c} / \mathbf{H z})\end{array}$ & $\begin{array}{c}\text { Pdc } \\
\mathbf{( m W )}\end{array}$ & $\begin{array}{c}\text { FOM}^{*} \\
(\mathbf{d B c} / \mathbf{H z})\end{array}$ & Ref. \\
\hline 89.2 & 10.2 & 8.0 & -112 & 650 & 162.8 & {$[6]$} \\
\hline 67 & 19.5 & 4.85 & -111 & 660 & 159.3 & {$[7]$} \\
\hline 71 & 19.3 & 1.37 & -103 & 1470 & 148.3 & {$[8]$} \\
\hline $\mathbf{8 4}$ & $\mathbf{- 0 . 6 7}$ & $\mathbf{1}$ & $\mathbf{- 1 2 0}$ & $\mathbf{3 4 0}$ & $\mathbf{1 7 1 . 1}$ & This \\
\hline
\end{tabular}

$* F O M(\Delta f)=-\mathcal{L}(\Delta f)+20 \times \log _{10}\left(f_{0} / \Delta f\right)-10 \times \log _{10}\left(P_{D C} / 1 m W\right)$

\section{CONCLUSION}

In this work, a low phase noise W-band MMIC GaN HEMT oscillator is reported. The design is based on common-gate balanced Colpitts topology and is fabricated in the advanced OMMIC's $60 \mathrm{~nm}$ GaN HEMT technology. Measured output power of nearly $0 \mathrm{dBm}$, and far-carrier phase noise of $-120 \mathrm{dBc} / \mathrm{Hz}$ at $10 \mathrm{MHz}$ offset, are reached. It is found that phase noise at $10 \mathrm{MHz}$ offset of the designed oscillator is state-of-the-art number for W-band MMIC GaN HEMT oscillators. The design shows the feasibility of low phase noise MMIC GaN HEMT oscillators operating at mmwave frequencies that can be used in mm-wave radars.

\section{ACKNOWLEDGMENT}

This work was financed by the CleanSky 2 program within the European Union's Horizon 2020 research and innovation framework, grant agreement no. 821270 "GaN mm-wave Radar Components Embedded - GRACE”.

\section{REFERENCES}

[1] S. Honma and N. Uehara, "Millimeter-Wave Radar technology for Automotive Application,” Technical reports, June 2001.

[2] M. Kishida, K. Ohguchi and M. Shono, "79 GHz-Band HighResolution Millimeter-Wave Radar,” FUJITSU Sci. Tech. J., vol. 51, no.4, pp. 55-59, Oct. 2015..

[3] H. Liu, X. Zhu, C. C. Boon, X. Yi, M. Mao and W. Yang, "Design of Ultra-Low Phase Noise and High Power Integrated Oscillator in 0.25 $\mu \mathrm{m}$ GaN-on-SiC HEMT technology,” IEEE Microw. Wireless Compon. Lett., vol.24, no. 2, pp. 120-122, 2015.

[4] H. Zirath, R. Kozhuharov and M. Ferndahl, "Balanced Colpittsoscillator MMICs designed for ultra-low phase noise,” IEEE J. SolidState Circuits, vol. 40, no. 10, pp. 2077-2086, 2005.

[5] https://cdn.rohdeschwarz.com/pws/dl downloads/dl common library/dl brochures a nd_datasheets/pdf_1/FSUP_dat-sw_en.pdf.

[6] R. Weber, D. Schwantuschke, P. Bruckner, R. Quay, F. Raay and O. Ambacher, “A $92 \mathrm{GHz}$ GaN HEMT Voltage-Controlled Oscillator MMIC,” IEEE. Inter. Microw. Symp. (IMS), Tampa, folrida, USA, 2014.

[7] R. Weber, D. Schwantuschke, P. Bruckner, R. Quay, M. Mikulla, O. Ambacher and I. Kallfass, " A 67 GHz GaN Voltage-Controlled Oscillator MMIC with high output power," IEEE Microw. Wireless Compon. Lett., vol. 23, no. 7, pp. 374- 376, Jul. 2013.

[8] Y. Nakasha, et. al., "E-band 85-mW Oscillator and 1.3-W Amplifier ICs using 0.12-llm GaN HEMTs for Millimeter-wave Transceivers," IEEE Compo Semic. Integr. Corc. Symp. (CSICS), Monterey, CA, USA, pp. 199-202, 2010. 